\title{
Regulatory effect of the MAFG-AS1/miR-150-5p/MYB axis on the proliferation and migration of breast cancer cells
}

\author{
HONGYAO JIA*, DI WU*, ZHIRU ZHANG and SIJIE LI \\ Department of Breast Surgery, The First Hospital of Jilin University, Changchun, Jilin 130021, P.R. China
}

Received December 25, 2019; Accepted August 25, 2020

DOI: 10.3892/ijo.2020.5150

\begin{abstract}
Long noncoding RNA (lncRNA) MAF BZIP transcription factor $\mathrm{G}$ antisense RNA 1 (MAFG-AS1) has been demonstrated to serve an important role in the progression of various types of cancer, whereas its role in breast cancer has not been fully elucidated. The present study aimed to explore the potential role and underlying mechanism of MAFG-AS1 in breast cancer. To achieve this, the expression of MAFG-AS1, microRNA (miR)-150-5p and MYB was detected by reverse transcription-quantitative PCR. The binding between miR-150-5p and MAFG-AS1 or MYB was verified using a luciferase reporter assay. Cell proliferation was analyzed by MTS, apoptosis and cell cycle were detected by Annexin V/propidium iodide, and cell migration was analyzed by wound healing assay. The results demonstrated that the expression levels of MAFG-AS1 were significantly upregulated in breast cancer tissues and cells compared with those in normal breast tissues and cells. High MAFG-AS1 expression promoted the proliferation, migration and epithelial-mesenchymal transition of breast cancer cells. By contrast, miR-150-5p expression was reduced in breast cancer tissues compared with that in healthy breast tissues, and low expression of miR-150-5p was associated with poor overall survival in patients with breast cancer. Bioinformatics and luciferase assay revealed that MAFG-AS1 served as a sponge of miR-150-5p, and that miR-150-5p bound to MYB. The functional rescue assay results demonstrated that MAFG-AS1 knockdown suppressed the proliferation and migration of breast cancer cells by regulating miR-150-5p, which in turn targeted MYB. In conclusion, the results of the present study demonstrated that MAFG-AS1 functioned as a novel oncogenic lncRNA in the development of human breast cancer via regulating the miR-150-5p/MYB axis, which
\end{abstract}

Correspondence to: Dr Sijie Li, Department of Breast Surgery, The First Hospital of Jilin University, 71 Xinmin, Chaoyang, Changchun, Jilin 130021, P.R. China

E-mail: lisij@jlu.edu.cn

*Contributed equally

Key words: long non-coding RNA MAFG-AS1, microRNA-150-5p, MYB, breast cancer suggested that MAFG-AS1 may be a novel biomarker for the diagnosis and prognosis of human breast cancer.

\section{Introduction}

Breast cancer is one of the most commonly diagnosed malignant cancers and is the second leading cause of cancer-related deaths among women worldwide (1). An estimated $36.1 \%$ incidence and 8.8\% mortality occurred in China in $2018(2,3)$. Although there are treatments for breast cancer, including individualized treatment, the pathogenesis of breast cancer has not yet been fully elucidated, leading to poor outcomes of patients. The 5-year survival rate of patients with breast cancer is usually $<30 \%$, which requires improvement (4). Therefore, there is an urgent need to further investigate the carcinogenesis and development of breast cancer.

Long non-coding RNAs (lncRNAs) are a large group of transcripts $>200$ nucleotides with limited protein-coding potential $(5,6)$. Studies have demonstrated that lncRNAs serve a range of specific functions in various pathophysiological processes $(6,7)$, especially in cancer $(8,9)$. For example, HOX transcript antisense RNA, an oncogenic lncRNA, is highly expressed in breast cancer, and its expression is associated with tumor invasiveness and migration $(10,11)$. Upregulation of lncRNA P21-associated non-coding RNA DNA damage-activated is associated with poor prognosis and accelerates cell proliferation in cervical cancer (12). The expression of lncRNA paternally expressed 10 is upregulated in esophageal cancer tissues compared with that in adjacent non-neoplastic tissues, and its depletion suppresses proliferation and invasion and facilitates apoptosis in esophageal cancer cells (13). lncRNAs have been recommended for use as indicators for tumor detection due to their key role in tumors and their stability in the serum and plasma $(14,15)$. IncRNA MAF BZIP transcription factor $\mathrm{G}$ antisense RNA 1 (MAFG-AS1) is abnormally expressed in various types of tumors and is involved in the regulation of cancer cell proliferation and migration (16-18). However, the potential mechanisms of MAFG-AS1 in breast cancer have not been elucidated.

MicroRNAs (miRNAs) are a group of endogenous non-coding RNAs 21-23 nucleotides long that are present in eukaryotes (19). Numerous studies have revealed that miRNAs participate in cancer progression. For example, miR-195 expression is low in head and neck squamous cell carcinoma tissues compared with that in normal tissues and is sponged 
by LINC00355 to affect cell viability, invasion, migration, the epithelial-mesenchymal transition (EMT) process and apoptosis of cancer stem cells (20). miR-7-5p inhibits the proliferation, migration, invasion and EMT process of non-small cell lung cancer by suppressing neuro-oncological ventral antigen 2 (21). miR-182 is sponged by prostate cancer gene expression marker 1 and is involved in the progression of cervical cancer (22). miR-150-5p has been reported to serve an essential role in several types of cancer (23-25). However, whether miR-150-5p exerts a role in breast cancer remains unknown.

The present study aimed to explore the role of MAFG-AS1 and its regulatory mechanisms in breast cancer, which might provide new targets for breast cancer treatment.

\section{Materials and methods}

GEPIA analysis. The GEPIA database (http://gepia.cancer-pku. $\mathrm{cn} /$ ) was used to analyze the expression of MAFG-AS1 in breast cancer tissues. The breast cancer dataset was selected, and the levels of MAFG-AS1 in breast cancer tissues $(n=1,085)$ and non-tumor tissues $(\mathrm{n}=291)$ were plotted.

Cell culture and clinical specimens. Breast cancer cells (MDA-MB-231, MCF-7 and MDA-MB-468) and the normal epithelial breast cell line MCF-10A were obtained from ATCC. The cells were cultured in RPMI-1640 (HyClone; Cytiva) supplemented with 10\% FBS (Gibco; Thermo Fisher Scientific, Inc.) at $37^{\circ} \mathrm{C}$ with $5 \% \mathrm{CO}_{2}$.

A total of 20 samples of breast cancer tissues (median age, 48 years; range, 37-65 years) and 15 samples of adjacent non-tumor tissues (3-5 $\mathrm{cm}$ distal to the edge of tumor) were obtained after resection and immediately frozen at $-80^{\circ} \mathrm{C}$ at the First Hospital of Jilin University (Changchun, China) between April 2018 and June 2019. Based on the Tumor-Node-Metastasis staging system (26), five cases were stage I, 11 cases were stage II, and four cases were stage III. The clinical study was approved by the Ethics Committee of the First Hospital of Jilin University. All patients participating in the study provided written informed consent. All procedures were performed according to the guidelines revised by the First Hospital of Jilin University and in accordance with the 1964 Declaration of Helsinki and its later amendments. The diagnoses were based on pathology reports. Patients with other tumors, tumor history or those who received chemotherapy and radiotherapy prior to the surgery were excluded.

Cell transfection. The MAFG-AS1 sequence and negative control were constructed by Shanghai GenePharma Co., Ltd. and cloned into the pcDNA3.1 vector (Shanghai GenePharma Co., Ltd.). The day before transfection, MCF-7 cells were seeded in 6-well plates at the confluency of 25-30\%. miRNA mimics, inhibitor, short hairpin (sh)RNA plasmids and the corresponding negative controls ( $\mathrm{NC}$ mimics, $\mathrm{NC}$ inhibitor and sh-NC) were purchased from Guangzhou RiboBio Co., Ltd. and were transfected at a final concentration of $50 \mathrm{nM}$ using Lipofectamine ${ }^{\circledR}$ RNAiMax (Invitrogen; Thermo Fisher Scientific, Inc.) according to the manufacturer's instructions. The sequences were as follows: miR-150-5p mimics, 5'-UCUCCCAACCCUUGUACCAGUG-3', NC mimics,
5'-UUCUCCGAACGUGUCACGUTT-3'; miR-150-5p inhibitor, 5'-CACUGGUCAUCCUUCGGAGA-3'; NC inhibitor, 5'-UUGUACUACACAAAAGUACUG-3'; sh-MAFG-AS1, 5'-TTATCTTCCTCCCGAGTCC-3'; and sh-NC, 5'-AATTCT CCGAACGTGTCACGT-3'. The breast cancer cells were transfected with the above plasmids for $72 \mathrm{~h}$ for subsequent functional experiments.

$R N A$ isolation, reverse transcription-quantitative ( $R T-q) P C R$. Total RNA was extracted from breast cancer tissues and cells using TRIzol ${ }^{\circledR}$ reagent (Invitrogen; Thermo Fisher Scientific, Inc.). RNA (500 ng) was reverse-transcribed into cDNA using a Reverse Transcription kit (Takara Bio, Inc.) for $5 \mathrm{~min}$ at $65^{\circ} \mathrm{C}$. The reverse transcription products were diluted 1:5 with sterile water and stored at $-20^{\circ} \mathrm{C}$. The subsequent PCR amplification was performed on an Mx3000P instrument (Agilent Technologies, Inc.) with SYBR ${ }^{\circledR}$ Premix Ex Taq II (Takara Biotechnology Co., Ltd.). The thermocycling conditions were as follows: Pre-denaturation at $95^{\circ} \mathrm{C}$ for $10 \mathrm{sec}$, followed by 40 cycles of denaturation at $95^{\circ} \mathrm{C}$ for $10 \mathrm{sec}$ and elongation at $60^{\circ} \mathrm{C}$ for $30 \mathrm{sec}$. GAPDH and U6 were used as internal controls, and the fold-changes were calculated using the $2^{-\Delta \Delta C q}$ method (27). The primer sequences used were as follows: MAFG-AS1 forward, 5'-CGTTCTTAGTTGGTGGAGCG-3' and reverse, 5'-CCGGACATCTAAGGGCATCA-3'; GAPDH forward, 5'-ATGGGACGATGCTGGTACTGA-3' and reverse, 5'-TGCTGACAACCTTGAGTGAAAT-3'; miR-150-5p stem-loop, 5'-GTCGTATCCAGTGCAGGGTCCGAGGTA TTCGCACTGGATACGACCCACTGG-3', forward, 5'-TCTC CCAACCCTTGTA-3' and reverse, 5'-GTGCAGGGTCCG AGGT-3'; U6 forward, 5'-CTCGCTTCGGCAGCACA-3' and reverse, 5'-AACGCTTCACGAATTTGCGT-3'; and MYB forward, 5'-ACAGATGGGCAGAAATCGCA-3' and reverse, 5'-GCTGGCTGGCTTTTGAAGAC-3'.

Western blotting analysis. Total cellular proteins from MCF-7 cells were extracted using RIPA lysis buffer (Beyotime Institute of Biotechnology). Protein concentration was determined using a bicinchoninic acid assay (Beyotime Institute of Biotechnology), and $10 \mu \mathrm{g}$ protein/lane was separated by $10 \%$ SDS-PAGE followed by transferring to polyvinylidene fluoride membranes (EMD Millipore). The membranes were blocked with 5\% non-fat milk for $1 \mathrm{~h}$ and incubated at $4^{\circ} \mathrm{C}$ with the following primary antibodies overnight: Anti-MYB (cat. no. ab191064; 1:1,000; BD Biosciences), anti-E-cadherin (cat. no. ab269767; 1:1,000; BD Biosciences), anti-N-cadherin (cat. no. ab18203; 1:1,000; BD Biosciences), anti-Bcl-2 (cat. no. ab194583; 1:1,000; BD Biosciences), anti-Bax (cat. no. ab104156; 1:1,000; BD Biosciences), anti-cyclin A1 (cat. no. ab53699; 1:1,000; BD Biosciences), anti-CDK2 (cat. no. ab64669; 1:1,000; BD Biosciences) and anti-GAPDH (cat. no. ab9485; 1:1,000; Affinity Biosciences). The membranes were washed three times using PBS, incubated with goat anti-rabbit IgG secondary antibody conjugated to horseradish peroxidase (cat. no. ab7090; 1:5,000; Abcam) for $1 \mathrm{~h}$ at room temperature and visualized using a Pierce ECL Western Blotting kit (Pierce; Thermo Fisher Scientific, Inc.). The protein expression was quantified using Image-Pro ${ }^{\circledR}$ Plus software (version 6.0; Media Cybernetics, Inc.). 
Subcellular fractionation. The cytoplasmic and nuclear RNA was isolated from MCF-7 cells using a PARIS kit (Invitrogen; Thermo Fisher Scientific, Inc.) according to the manufacturer's instructions. The cells were lysed in the cell fractionation buffer, followed by centrifugation for $5 \mathrm{~min}$ at $500 \mathrm{x} \mathrm{g}$ at $4^{\circ} \mathrm{C}$ and lysis in cell disruption buffer. RT-qPCR was used to determine the expression of MAFG-AS1 in both fractions as aforementioned, with GAPDH and U6 as cytoplasmic and nuclear controls, respectively.

Luciferase reporter assay. The binding site between miR-150-5p and MAFG-AS1 was predicted using the starBase website (28). The fragment of MYB or MAFG-AS1 containing the specific binding sites of miR-150-5p was transfected into the pmirGLO Dual-luciferase vector (Promega Corporation) to construct the reporter vectors containing wild-type MYB (WT-MYB) or wild-type MAFG-AS1 (WT-MAFG-AS1) 3' untranslated region (3'UTR). Three online algorithms miRDB (http://mirdb.org/), TargetScan (http://www.targetscan. org/vert_72/) and miRanda (http://www.miranda.org/) were used to predict the potential target genes of miR-150-5p. The mutated MYB (Mut-MYB) or mutated MAFG-AS1 (Mut-MAFG-AS1) vectors contained mutated binding sites. MCF-7 cells were co-transfected with WT-MAFG-AS1/MYB or Mut-MAFG-AS1/MYB and miR-150-5p mimics using Lipofectamine ${ }^{\circledR}$ RNAiMax for $48 \mathrm{~h}$ at $22^{\circ} \mathrm{C}$. The luciferase activity was detected using the Dual-Luciferase Reporter Assay System (Promega Corporation). Renilla luciferase activity was used to normalize the firefly luciferase activity.

Cell viability detection assay. After transfection with the indicated plasmids, MCF-7 cells $\left(2 \times 10^{3}\right.$ cells/well) were re-seeded on 96-well plates. The viability of breast cancer cells was assessed by the MTS method (Promega Corporation) at daily intervals for 3 days post-transfection. At each time point, MTS was added to the corresponding plates and then incubated for $2 \mathrm{~h}$ at $37^{\circ} \mathrm{C}$ according to the manufacturer's instructions. The optical density values were measured at $490 \mathrm{~nm}$ using a Multiskan EX microplate reader (Labsystems Diagnostics Oy).

Wound healing assay. MCF-7 were reseeded in a new 6-well plate $\left(\sim 5 \times 10^{5}\right.$ cells/well $)$ after transfection for $24 \mathrm{~h}$ and cultured to $80-90 \%$ confluence. Subsequently, a $200-\mu 1$ sterile pipette tip was used to generate a straight scratch. The cells were cultured in serum-free medium in a humidified incubator at $37^{\circ} \mathrm{C}$ with $5 \% \mathrm{CO}_{2}$. Finally, the migration distance was observed, and images were captured at $0 \mathrm{~h}$ and $24 \mathrm{~h}$ post-scratch. The scratch area was recorded by a light microscope (Olympus Corporation) and analyzed with ImageJ version 1.47 software (National Institutes of Health). The distance of cell migration into the wound was measured to calculate the wound healing rate as follows: Wound healing rate $(\%)=($ wound distance at $0 \mathrm{~h}$-wound distance at $24 \mathrm{~h}$ )/wound distance at $0 \mathrm{~h} \times 100 \%$.

Cell cycle and apoptosis analysis. MCF-7 cells $\left(1 \times 10^{6}\right.$ cells/well) were fixed with $70 \%$ pre-cooled ethanol overnight at $4^{\circ} \mathrm{C}$, treated with RNase A (Sigma-Aldrich; Merck $\mathrm{KGaA}$ ) at $37^{\circ} \mathrm{C}$ for $30 \mathrm{~min}$ and stained with propidium iodide (PI) (Nanjing Keygen Biotech Co., Ltd.) staining solution at $4^{\circ} \mathrm{C}$ for 30 min following transfection with miRNA mimics. Subsequently, each group of cells was tested using a BD FACSCalibur flow cytometer (BD Biosciences), and the results were analyzed using FlowJo7.6 software (FlowJo Software, LLC). For apoptosis, an Annexin V-FITC/propidium iodide (PI) staining kit (BD Biosciences) was used. Briefly, MCF-7 cells suspended in $100 \mu \mathrm{l}$ binding buffer $\left(1 \times 10^{5}\right.$ cells $\left./ \mathrm{ml}\right)$ were incubated at room temperature for $15 \mathrm{~min}$ in the dark with $5 \mu \mathrm{l}$ Annexin V-FITC and $5 \mu \mathrm{l}$ PI solution. The apoptotic cells were quantified using a BD FACSCalibur flow cytometer, and the data were analyzed with BD Accuri ${ }^{\mathrm{TM}}$ C6 software (BD Biosciences). The apoptotic rate was calculated using the following formula: Apoptotic rate=(early apoptotic cells + late apoptotic cells)/total cell number $\times 100 \%$.

Xenograft study. A total of 50 ( $\mathrm{n}=6-8 \mathrm{mice} / \mathrm{group})$ female BALB/C nude mice at age of 6-8 weeks were obtained from Beijing Vital River Laboratory Animal Technology Co., Ltd. The animal experiments were approved by the Ethics Committee of Animal Experiments of the First Hospital of Jilin University (approval no. DWLL-2019-0021). All mice were housed in a 12 -h light/dark cycle at $22^{\circ} \mathrm{C}$ with $40-70 \%$ humidity for at least 7 days before the experiments. Mozart K448 sonata was used to alleviate fear and stress, and the mice had free access to water and food. Animal health and behavior was monitored every day. The mice were randomly divided into five groups: pcDNA + NC mimics; MAFG-AS1 + NC mimics; MAFG-AS1 + miR-150-5p mimics; pcDNA + miR-150-5p mimics; and miR-150-5p mimics + MYB. MCF-7 cells $\left(5 \times 10^{6}\right)$ suspended in $150 \mu \mathrm{l}$ PBS were subcutaneously injected into the right armpit of mice. At 21 days post-inoculation, the mice were sacrificed by cervical dislocation. The xenograft tumors were carefully excised from the sacrificed mice and weighted, and the tumor volume was calculated using the following formula: Volume $=0.5 \mathrm{x}$ length $\mathrm{x}$ width ${ }^{2}$.

Statistical analysis. Data are presented as the mean \pm standard deviation. All experimental data were processed and analyzed using SPSS 22.0 statistical software (IBM Corp.). The Kaplan-Meier plotter database (http://kmplot.com/analysis/) was used to analyze the association between miR-150-5p expression and overall survival (OS) of patients with breast cancer. The total number of patients was 1,262 , and based on the median expression of miR-150, the patients were divided into high and low expression groups. The Kaplan-Meier method was used for survival analysis, and the log-rank test was performed compare the two groups. Differences between two groups were evaluated by two-tailed Student's t-test. Statistical significance among three or more groups was assessed by one- or two-way ANOVA followed by Dunnett's (for comparisons with one control) or Tukey's (for comparisons among various groups) post hoc test. $\mathrm{P}<0.05$ was considered to indicate a statistically significant difference.

\section{Results}

$M A F G-A S 1$ is upregulated in breast carcinoma. In the present study, the expression levels of MAFG-AS1 were compared between breast cancer and adjacent non-tumor tissues from patients using original published data available from the 
A

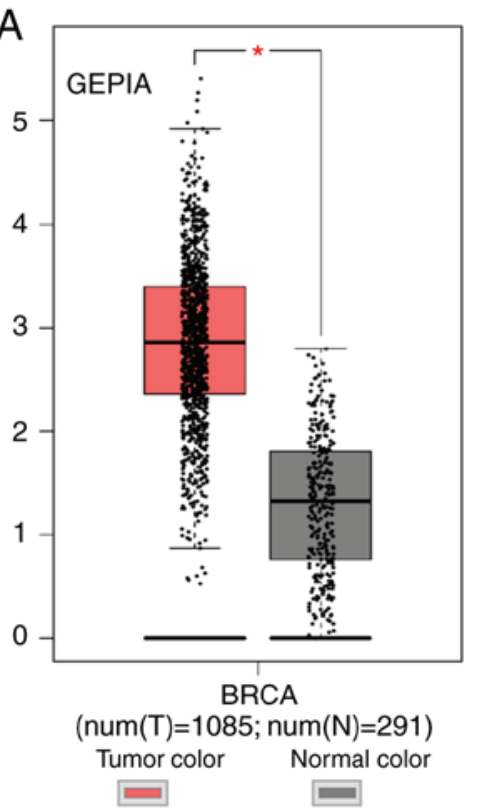

B

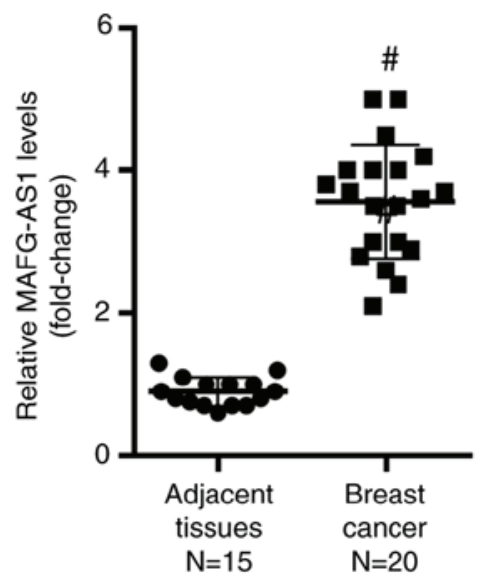

C

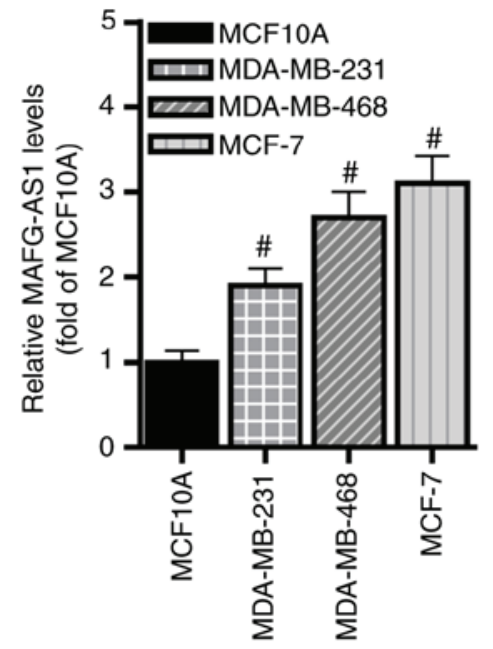

Figure 1. MAFG-AS1 is upregulated in breast cancer tissues and cells. (A) GEPIA database analysis revealed high expression in MAFG-AS1 breast cancer tissues. ${ }^{*} \mathrm{P}<0.05$ vs. normal tissues. (B) Reverse transcription-quantitative PCR analysis demonstrated that the expression levels of MAFG-AS1 were upregulated in breast cancer tissues compared with those in adjacent non-tumor tissues. ${ }^{\#} \mathrm{P}<0.05$ vs. adjacent tissues. (C) MAFG-AS1 expression levels were upregulated in three breast cancer cell lines compared with those in the normal breast epithelial cell line MCF10A. ${ }^{\text {P }<0.05 ~ v s . ~ M C F 10 A . ~ M A F G-A S 1, ~ M A F ~}$ BZIP transcription factor $\mathrm{G}$ antisense RNA 1 .

GEPIA database. The results demonstrated that the expression MAFG-AS1 was significantly upregulated in breast cancer samples compared with that in non-tumor samples (Fig. 1A). In addition, RT-qPCR revealed that MAFG-AS1 expression levels were higher in cancer tissues compared with those in adjacent non-tumor tissues (Fig. 1B). Similarly, MAFG-AS1 levels were significantly upregulated in three human breast cancer cell lines (MDA-MB-468, MDA-MB-231 and MCF-7) compared with those in the normal breast epithelial cell line MCF-10A (Fig. 1C). These results suggested that MAFG-AS1 may function as a tumor-promoting lncRNA in breast cancer.

$M A F G-A S 1$ sponges miR-150-5p. Considering that the competing endogenous RNA (ceRNA) hypothesis, which indicates that lncRNAs can sponge miRNAs to upregulate the expression of specific mRNAs and further regulate the progression of diseases (29), is currently widely accepted, we hypothesized that MAFG-AS1 may also function in this manner in breast cancer. Cytoplasmic lncRNAs have been reported to present with a high probability of acting as ceRNAs (30); thus, cytoplasmic and nuclear MAFG-AS1 were extracted separately, and RT-qPCR was used to analyze the expression of MAFG-AS1 in the nucleus and cytoplasm of breast cancer cells. The results demonstrated that MAFG-AS1 was mainly concentrated in the cytoplasm of breast cancer cells (Fig. 2A), suggesting that MAFG-AS1 may be involved in a ceRNA network in breast cancer. To identify the potential mechanism of MAFG-AS1 in the modulation of the development of breast cancer, the miRNAs sponged by MAFG-AS1 were predicted using the online bioinformatics analysis tool starBase (28). The results revealed that MAFG-AS1 bound miR-150-5p (Fig. 2B). To overexpress miR-150-5p, transfection of the miR-150-5p mimics into breast cancer cells was used (Fig. S1A). The subsequent luciferase reporter assay results demonstrated that the luciferase activity of MCF-7 cells was significantly decreased by miR-150-5p mimics compared with that in the NC mimics group following transfection with WT-MAFG-AS1, whereas miR-150-5p mimics exerted no inhibitory effects on the luciferase activity of MCF-7 cells transfected with Mut-MAFG-AS1 (Fig. 2C). Taken together, these results indicated that MAFG-AS1 sponged miR-150-5p. The relationship between MAFG-AS1 and miR-150-5p was then further examined; as presented in Fig. 2D, transfection with the miR-150-5p mimics inhibited the expression of MAFG-AS1 in MCF-7 and MDA-MB-468 cells compared with the NC mimics group. Next, overexpression efficiency of MAFG-AS1 was verified by RT-qPCR analysis (Fig. S1B). Similarly, the expression of miR-150-5p was downregulated following overexpression of MAFG-AS1 in breast cancer cells compared with that in cells transfected with the empty vector (Fig. 2E). In addition, the mean expression levels of miR-150-5p were significantly reduced in cancer tissues compared with those in the adjacent non-tumor tissues (Fig. 2F). The association between miR-150-5p expression and OS of patients with breast cancer was analyzed using the Kaplan-Meier plotter database. Based on the median miR-150 expression, the patients were divided into high and low expression groups. The OS time of patients with high miR-150-5p expression was significantly longer compared with that of patients with low miR-150-5p expression (Fig. 2G). In conclusion, these results demonstrated that MAFG-AS1 interacted with miR-150-5p in breast cancer, and that high expression levels of miR-150-5p was associated with a favorable prognosis in patients with breast cancer. 


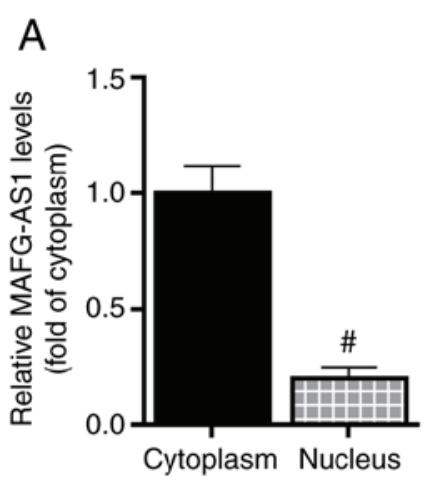

D

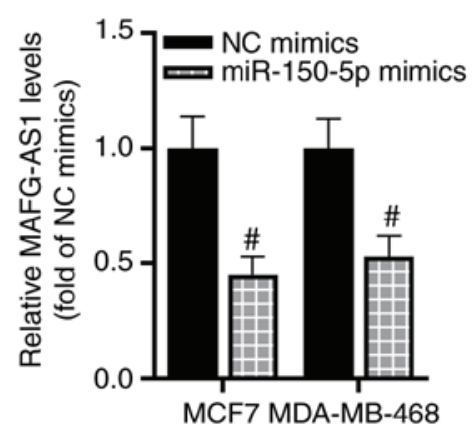

Binding sites of MAFG-AS1 with miR-150-5p

MAFG-AS1 Mut

MAFG-AS1 WT AUCCCAG U G CAG

CACU UGGAGG UGGGAGA

||| | - - || | |||||| |

miR-150-5p

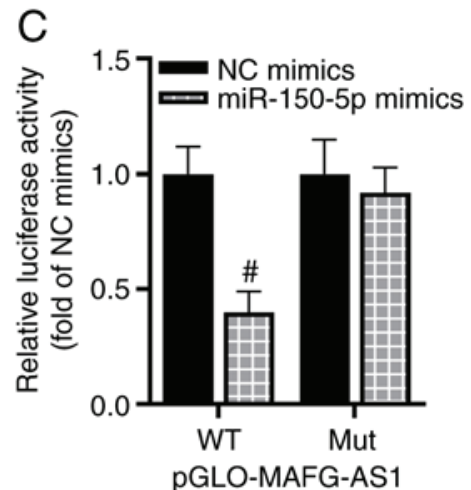

$E$

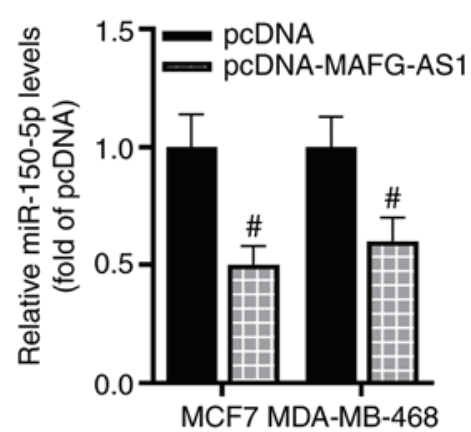

hsa-miR-150

F

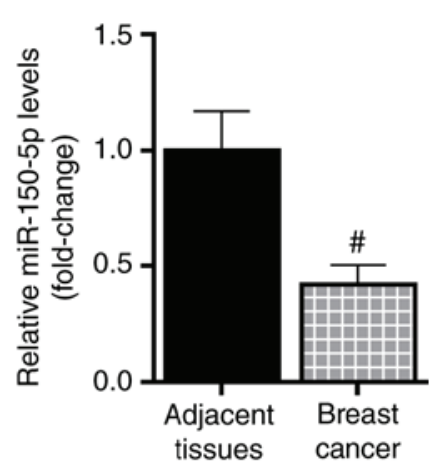

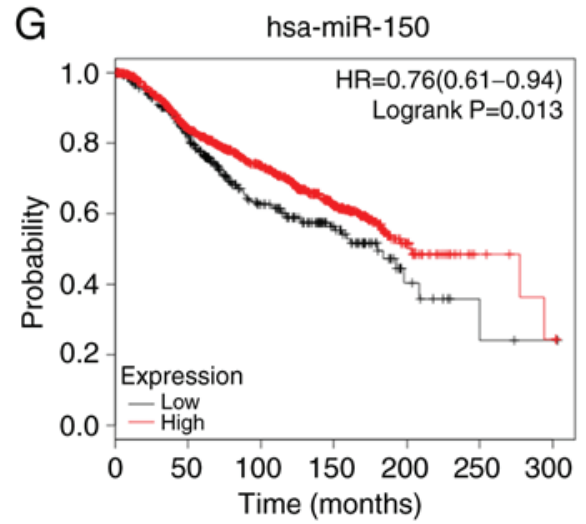

Figure 2. MAFG-AS1 sponges miR-150-5p. (A) Subcellular fractionation of the expression of MAFG-AS1 in the nucleus and cytoplasm of MCF-7 cells. ${ }^{\text {\#P}} \mathrm{P} 0.05$ vs. cytoplasm. (B) Target binding between MAFG-AS1 and miR-150-5p. Red represents mutated bases. (C) The interaction between miR-150-5p and MAFG-AS1 was confirmed by luciferase reporter assay. ${ }^{\prime} \mathrm{P}<0.05$ vs. NC mimics + WT-pGLO-MAFG-AS1. (D) The expression of MAFG-AS1 in MCF-7 and MDA-MB-468 cells transfected with miR-150-5p was determined by RT-qPCR. ${ }^{*} \mathrm{P}<0.05$ vs. NC mimics. (E) The expression of miR-150-5p in MCF-7 and MDA-MB-468 cells transfected with pcDNA-MAFG-AS1 or pcDNA was determined by RT-qPCR. "P<0.05 vs. pcDNA. (F) RT-qPCR was used to detect the expression of miR-150-5p in breast cancer and adjacent tissues. ${ }^{*} \mathrm{P}<0.05$ vs. adjacent tissues. (G) The Kaplan-Meier plotter database was used to analyze the association between miR-150-5p expression and overall survival of patients with breast cancer. Log-rank test was performed to analyze the comparisons between the groups. $\mathrm{n}=1,262 ; \mathrm{P}=0.013$. MAFG-AS1, MAF BZIP transcription factor G antisense RNA 1; miR, microRNA; WT, wild-type; Mut, mutant; RT-qPCR, reverse transcription-quantitative PCR.

MAFG-AS1/miR-150-5p regulates the viability and migration of breast cancer cells. The biological role of the MAFG-AS1/miR-150-5p axis in the development of breast cancer was further investigated. As presented in Fig. 3A, compared with that in the pcDNA + NC mimics group, overexpression of MAFG-AS1 significantly promoted the viability of breast cancer cells, whereas the miR-150-5p mimics reduced cell viability after $48 \mathrm{~h}$. In addition, the results demonstrated that co-transfection with the miR-150-5p mimics significantly abolished the increase in cell viability induced by MAFG-AS1 overexpression. The cell cycle analysis demonstrated that MCF-7 cells accumulated in the S phase and were decreased in the G1/G0 phase after pcDNA-MAFG-AS1 transfection, whereas the miR-150-5p mimics induced cell cycle arrest at the G1/G0 phase. However, the miR-150-5p mimics failed to induce G1/G0 arrest in pcDNA-MAFG-AS1 co-transfected MCF-7 cells (Fig. 3B). RT-qPCR was conducted to determine the expression of the cell cycle-associated genes cyclin A1 and CDK2. These results demonstrated that the expression levels of cyclin A1 and CDK2 were significantly upregulated in MCF-7 cells transfected with the pcDNA-MAFG-AS1 vector, but downregulated by the miR-150-5p mimics compared with the pcDNA + NC mimics group (Fig. 3C). A wound healing assay was conducted to assess the migratory ability 

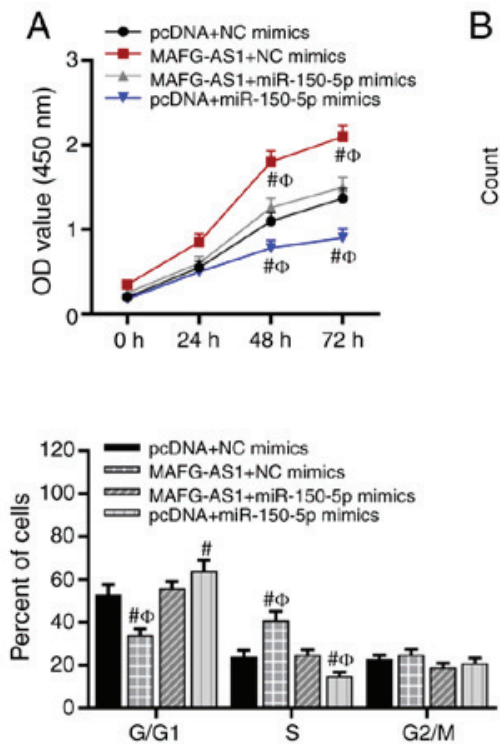

E
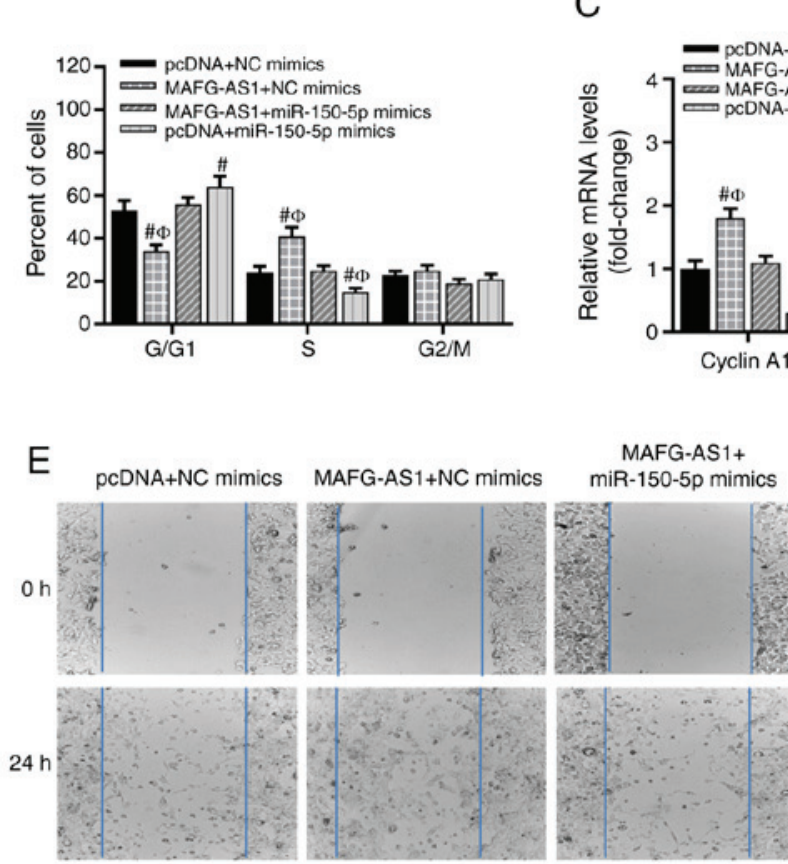

G
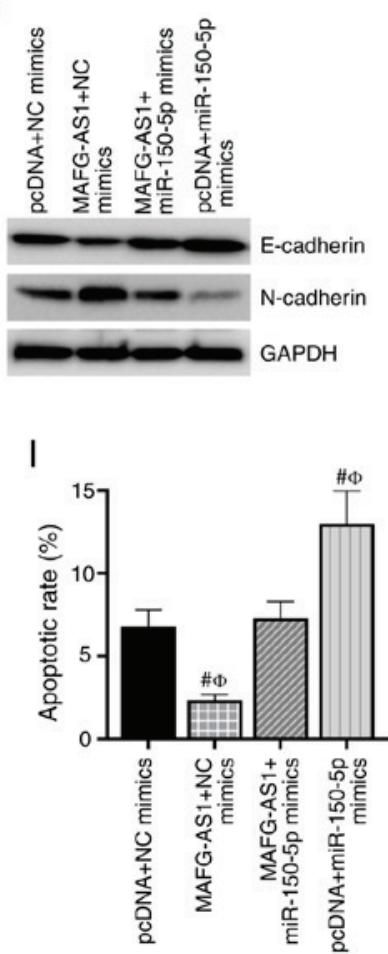

H

pcDNA+
- $150-5 p$ mimic

$\bar{\alpha}$

J

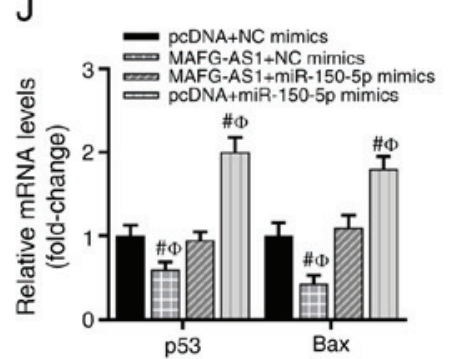

K

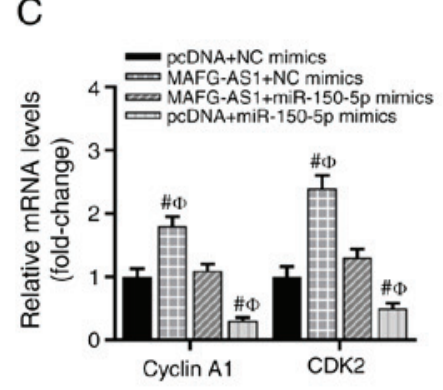

B

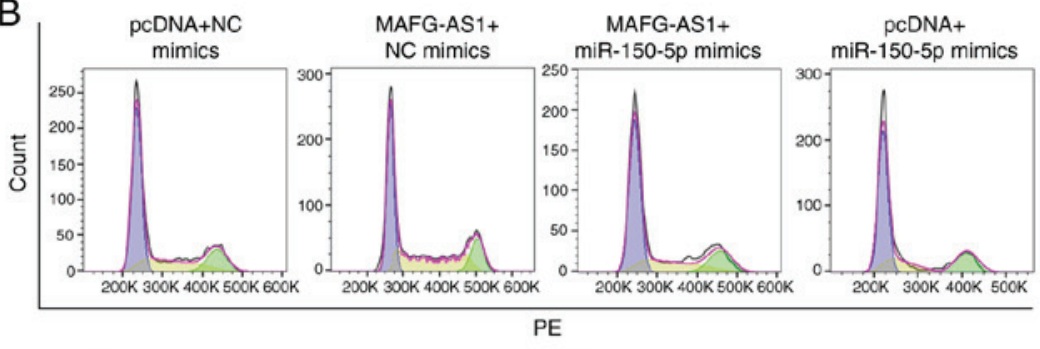

D
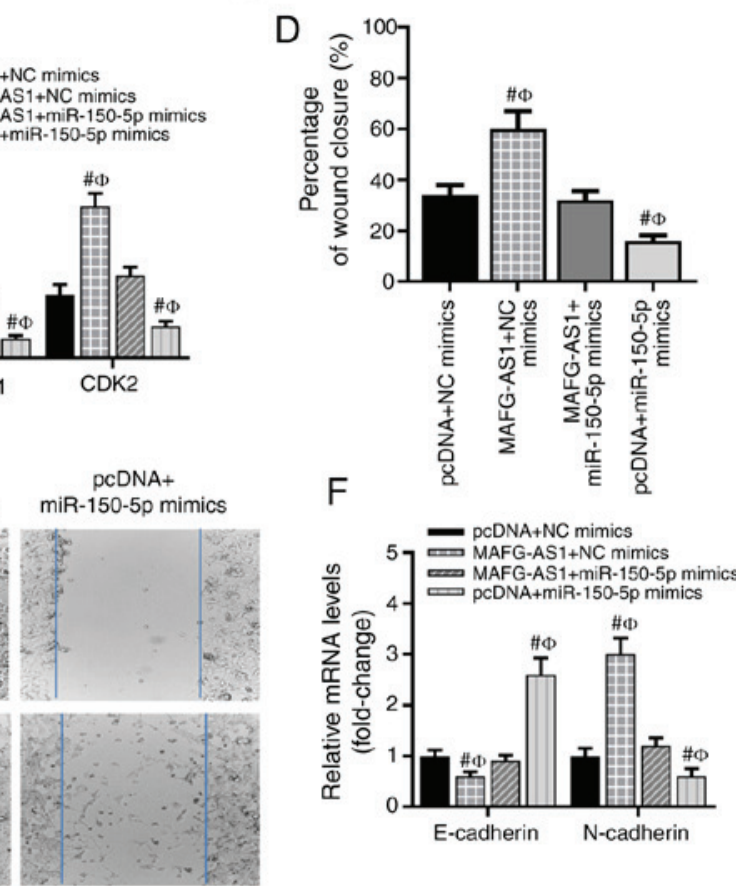
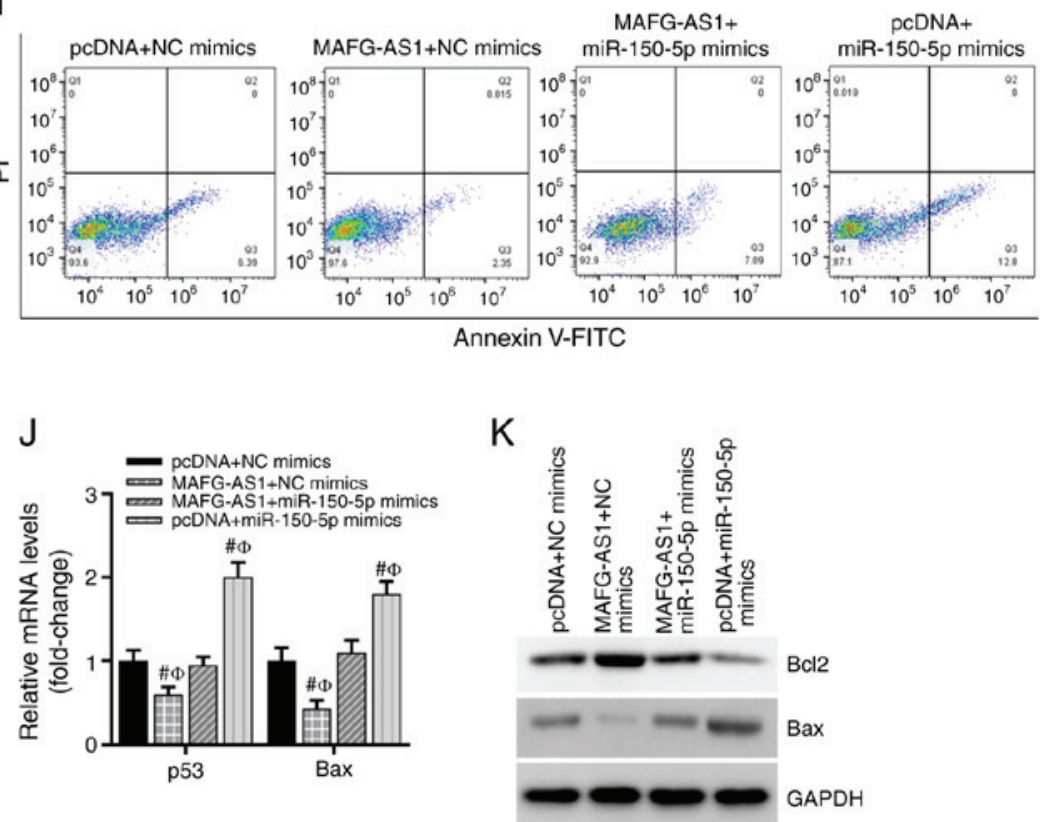

Figure 3. MAFG-AS1 and miR-150-5p regulate the biological functions of breast cancer cells. (A) MTS was used to detect cell proliferation in each group. (B) The cell cycle was examined using FACS analysis, and the cell cycle distribution was quantified. (C) RT-qPCR was used to detect the expression of cyclin A1 and CDK2. (D and E) Wound healing assay was used to determine the migratory ability of cells in each group, and statistical analysis was performed (F and G) RT-qPCR was used to assess the mRNA expression levels of E-cadherin and N-cadherin. (H) Representative flow cytometry images of apoptosis analysis. (I) The percentage of apoptotic cells. (J) The mRNA and (K) protein expression of the apoptotic genes Bcl-2 and Bax were detected by RT-qPCR and western blotting analysis, respectively, in each group of cells. ${ }^{*} \mathrm{P}<0.05$ vs. pcDNA + NC mimics; ${ }^{\Phi} \mathrm{P}<0.05$ vs. MAFG-AS1 + miR105-5p. MAFG-AS1, MAF BZIP transcription factor G antisense RNA 1; miR, microRNA; RT-qPCR, reverse transcription-quantitative PCR. 
of breast cancer cells. The results revealed that transfection with the miR-150-5p mimics inhibited, whereas overexpression of MAFG-AS1 promoted cell migration compared with that in the control group; in addition, the promotive effect of MAFG-AS1 overexpression on cell migration was attenuated by the miR-150-5p mimics (Fig. 3D and E). EMT-related gene expression was subsequently assessed. The mRNA and protein expression levels of E-cadherin were significantly downregulated, and those of $\mathrm{N}$-cadherin were upregulated in breast cancer cells transfected with pcDNA-MAFG-AS1 vectors compared with those in the control cells, whereas co-transfection with the miR-150-5p mimics reversed the effects of MAFG-AS1 on the expression of these markers (Fig. 3F and G). Additionally, overexpression of MAFG-AS1 decreased the apoptotic rates of breast cancer cells as measured by flow cytometry with PI and Annexin V staining, whereas the numbers of apoptotic cells were markedly increased by the miR-150-5p mimics (Fig. $3 \mathrm{H}$ and I). The inhibitory role of MAFG-AS1 overexpression on apoptosis was reversed by the miR-150-5p mimics (Fig. 3H and I). In addition, the mRNA and protein levels of the apoptosis-related gene Bcl-2 was significantly upregulated, whereas those of Bax was significantly downregulated in breast cancer cells transfected with pcDNA-MAFG-AS1 vectors compared with those in the control cells (Fig. 3J and K). By contrast, the miR-150-5p mimics decreased the mRNA and protein levels of Bcl-2, but increased those of Bax; however, the miR-150-5p mimics failed to induce Bcl-2 downregulation and Bax upregulation in MAFG-AS1 vector co-transfected MCF-7 cells (Fig. 3J and K). These results suggested that the MAFG-AS1/miR-150-5p axis may regulate the viability, apoptosis and migration of breast cancer cells.

$M Y B$ is a functional target gene of miR-150-5p. Subsequently, the downstream target of miR-150-5p was explored. Three online algorithms (miRDB, TargetScan and miRanda) were used to predict the potential target genes of miR-150-5p. The results revealed that MYB was a candidate target gene of miR-150-5p (Fig. 4A). The miR-150-5p inhibitor was used to knock down miR-150-5p expression, and the knockdown efficiency was validated by RT-qPCR (Fig. S1C). As presented in Fig. 4B, the luciferase activity was significantly weakened by the miR-150-5p mimics compared with the NC mimics, but exhibited no changes when the 3'UTR binding sites were mutated. Co-transfection of the miR-150-5p inhibitor and the WT-MYB 3'UTR plasmid resulted in a significant increase in the luciferase signal. Additionally, MYB mRNA levels were downregulated following transfection with the miR-150-5p mimics, but upregulated when miR-150-5p expression was inhibited (Fig. 4C). Similar results were obtained for MYB protein expression by western blotting (Fig. 4D). In addition, compared with adjacent normal breast tissues, MYB mRNA expression was upregulated in breast cancer tissues (Fig. 4E). For further experiments, a MYB overexpression vector was used, and the transfection efficiency of MYB was verified by RT-qPCR (Fig. S1D). The MTS assay demonstrated that MYB overexpression reversed the suppressive effect of the miR-150-5p mimics on MCF-7 cell viability (Fig. 4F). In addition, overexpression of MYB inhibited the miR-150-5p-induced cell cycle arrest (Fig. 4G). The miR-150-5p mimics significantly inhibited cyclin A1, CDK2 and N-cadherin
mRNA and protein expression levels and increased the expression levels of E-cadherin compared with those in the control cells; following co-transfection with the MYB overexpression vector, these results were reversed (Fig. 4H-K). Additionally, the miR-150-5p mimics promoted apoptosis in breast cancer cells, whereas the number of apoptotic cells was recovered following MYB overexpression (Fig. 4L and M). The decreases in Bcl-2 and increases in Bax expression levels induced by the miR-150-5p mimics were reversed by MYB overexpression (Fig. 4N and O). Overall, these results demonstrated that MYB was a functional target gene of miR-150-5p in breast cancer.

$M A F G$-AS1 positively regulates the expression of the MYB gene by sponging miR-150-5p. To confirm the relationship between MAFG-AS1/miR-150-5p and MYB expression, a luciferase reporter assay was performed. As presented in Fig. 5A, the MYB-WT luciferase activity was significantly increased by MAFG-AS1 overexpression and decreased by the miR-150-5p mimics. However, induction of luciferase activity of MYB-WT was abrogated following co-transfection with the MAFG-AS1 overexpression vector and the miR-150-5p mimics. The effects of MAFG-AS1/miR150-5p on MYB mRNA and protein expression were also assessed; RT-qPCR and western blotting results demonstrated that MAFG-AS1 overexpression upregulated the levels of MYB mRNA and protein expression, whereas transfection with the miR-150-5p mimics downregulated MYB mRNA and protein expression levels. Furthermore, MAFG-AS1 overexpression attenuated the inhibitory effects of the miR-150-5p mimics on the mRNA and protein expression of MYB (Fig. 5B-D). These results indicated that MAFG-AS1 competitively bound miR-150-5p to upregulate the expression of MYB.

$M Y B$ is a downstream molecule of MAFG-AS1 in the regulation of cell biological functions. To further validate the effects of MYB on the role of MAFG-AS1 in breast cancer cells, MAFG-AS1 expression was knocked down by transfection of shMAFG-AS1 into MCF-7 cells, and the knockdown efficiency of MAFG-AS1 was validated by RT-qPCR analysis (Fig. S1E). The MTS assay results demonstrated that compared with that in the pcDNA group, cell proliferation was significantly inhibited after silencing MAFG-AS1 expression but restored following co-transfection with the MYB overexpression vector (Fig. 6A). In addition, knockdown of MAFG-AS1 induced breast cancer cell cycle arrest in the G0/G1 phase and reduced the number of cells in the $\mathrm{S}$ phase compared with that in the cells transfected with the control vector. The effects of shMAFG-AS1 were reversed following co-transfection with the MYB overexpression vector (Fig. 6B). The expression levels of cyclin A1 and CDK2 were downregulated after silencing MAFG-AS1 compared with those in the control cells, whereas overexpression of MYB restored their expression (Fig. 6C). Similarly, the expression levels of the EMT-related protein E-cadherin were upregulated and those of $\mathrm{N}$-cadherin were downregulated in the shMAFG-AS1 group compared with those in the control group, and overexpression of MYB restored their expression (Fig. 6D and E). As presented in Fig. 6F and G, compared with that in the control group, the apoptotic rate was increased in the shMAFG-AS1 group, but decreased following co-transfection with the MYB overexpression vector. The mRNA and protein 
A

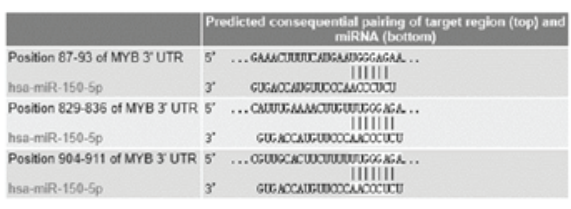

D

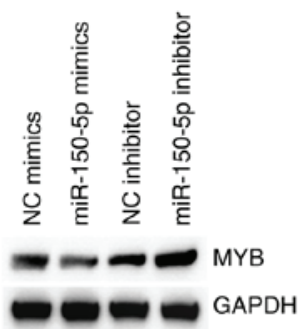

E

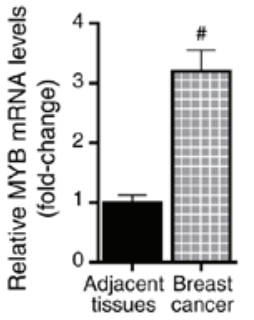

B

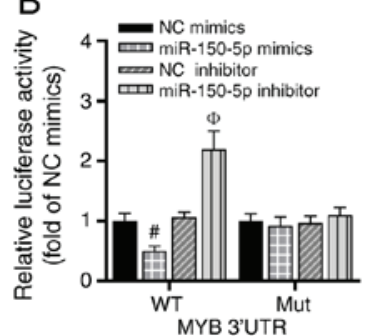

$\mathrm{F}$

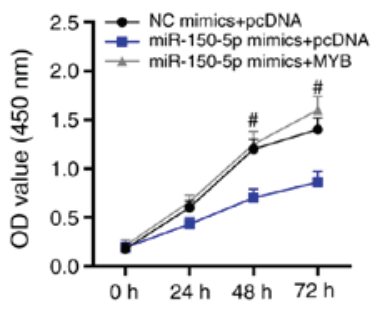

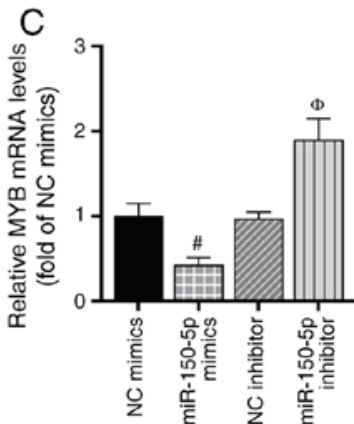

G

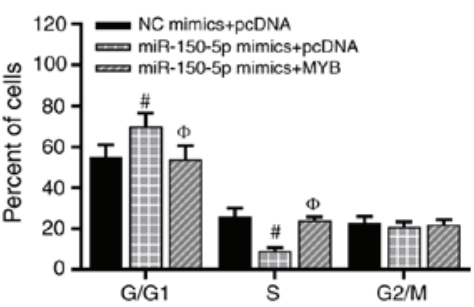

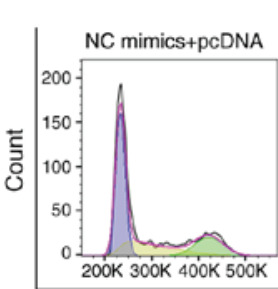

miR-150-5p mimics+ pcDNA
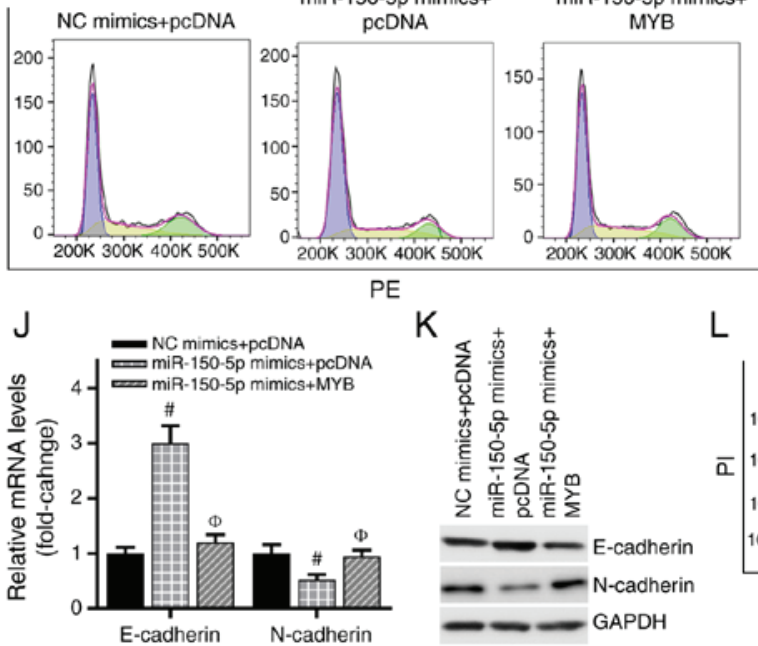

$\mathrm{K}$

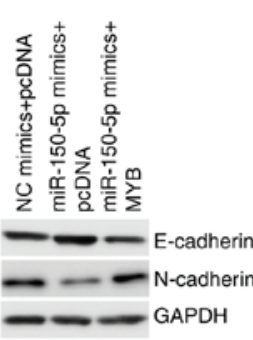

M

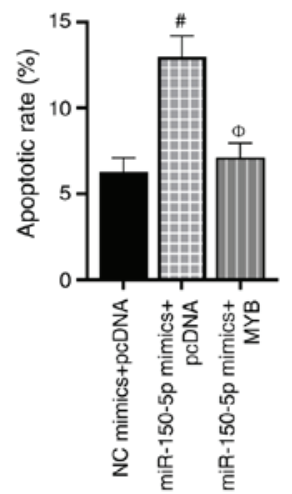

$\mathrm{H}$
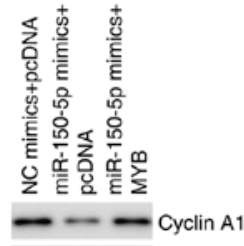

- - CDK2

$\longrightarrow$ GAPDH

L

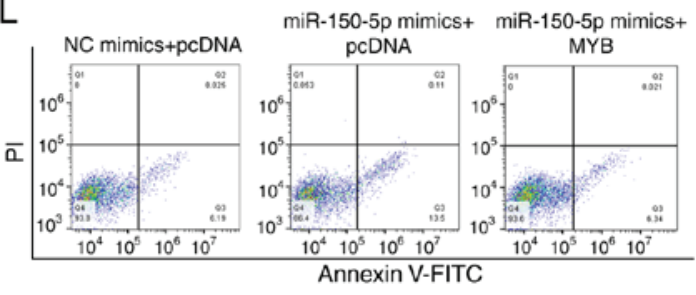

$\mathrm{N}$ 原常 $\mathrm{O}$

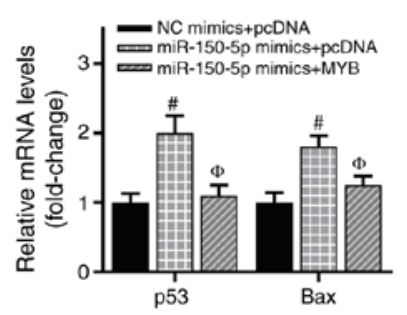

Figure 4. MYB is directly targeted by miR-150-5p. (A) Predicted miR-150-5p target sites in the MYB 3'UTR. Lines indicate perfect matches. Mut represents a mutation of $\mathrm{G}$ to $\mathrm{C}$ in the MYB 3'UTR. (B) Relative luciferase activity was detected by luciferase reporter assay. ${ }^{\#} \mathrm{P}<0.05$ vs. $\mathrm{NC}$ mimics; ${ }^{\Phi} \mathrm{P}<0.05$ vs. $\mathrm{NC}$ inhibitor. (C) RT-qPCR assay was used to detect MYB mRNA expression in each group of cells. ${ }^{\#} \mathrm{P}<0.05$ vs. NC mimics; ${ }^{\oplus} \mathrm{P}<0.05$ vs. NC inhibitor. (D) $\mathrm{MYB}$ protein expression was determined by western blotting analysis. (E) The expression of MYB mRNA in clinical tissue samples was confirmed by RT-qPCR. ${ }^{\#} \mathrm{P}<0.05$ vs. adjacent tissues. (F) MTS was used to detect cell proliferation of cells transfected with the miR-150-5p mimics and a MYB overexpression vector. ${ }^{\#} \mathrm{P}<0.05$ vs. miR-150-5p mimics + pcDNA. (G) The cell cycle was examined using FACS analysis, and the cell cycle distribution was quantified. (H) RT-qPCR was used to detect the expression of cyclin A1 and CDK2. (I) Western blotting analysis was used to detect the protein expression of cyclin A1 and CDK2. (J) The mRNA level of E-cadherin and N-cadherin in MCF-7 cells were detected by RT-qPCR. (K) The protein level of E-cadherin and N-cadherin in MCF-7 cells were detected by western blotting analysis. (L and M) Annexin V-FITC was used to detect the percentage of apoptotic cells in each group. (N) RT-qPCR was used to detect the expression of the Bcl-2 and Bax mRNA. (O) Western blotting analysis was used to detect the protein levels of the Bcl-2 and Bax. ${ }^{\#} \mathrm{P}<0.05$ vs. NC mimics + pcDNA; ${ }^{\Phi} \mathrm{P}<0.05$ vs. miR-150-5p mimics + pcDNA group. miR, microRNA; UTR, untranslated region; RT-qPCR, reverse transcription-quantitative PCR; OD, optical density. 
A
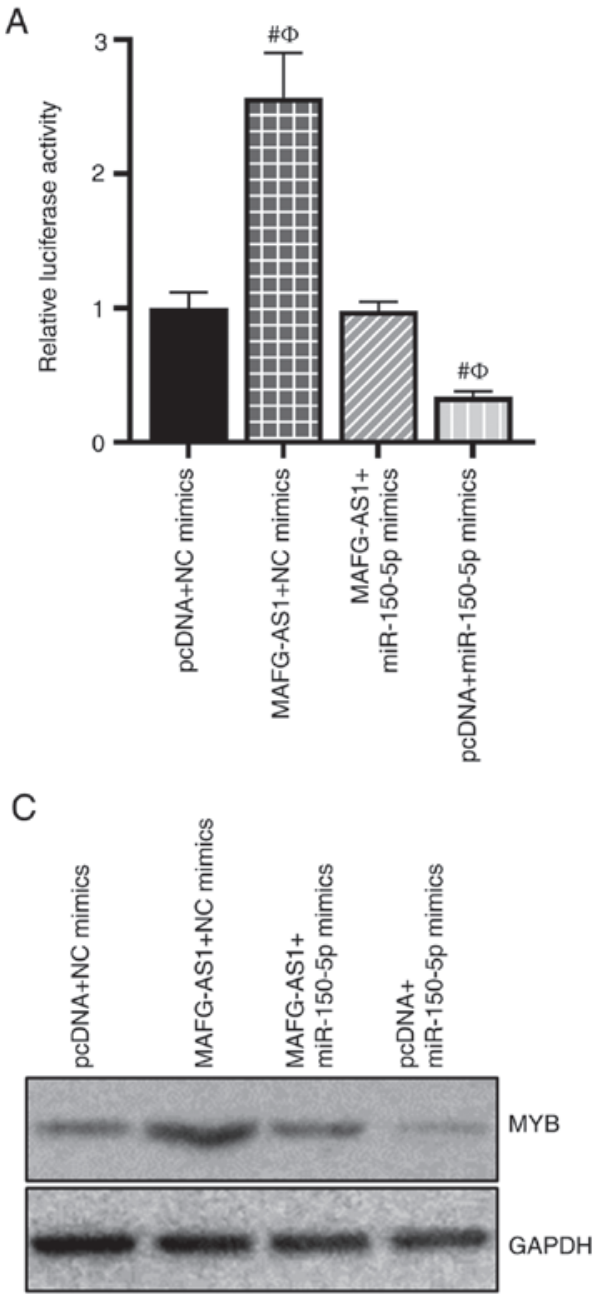

B
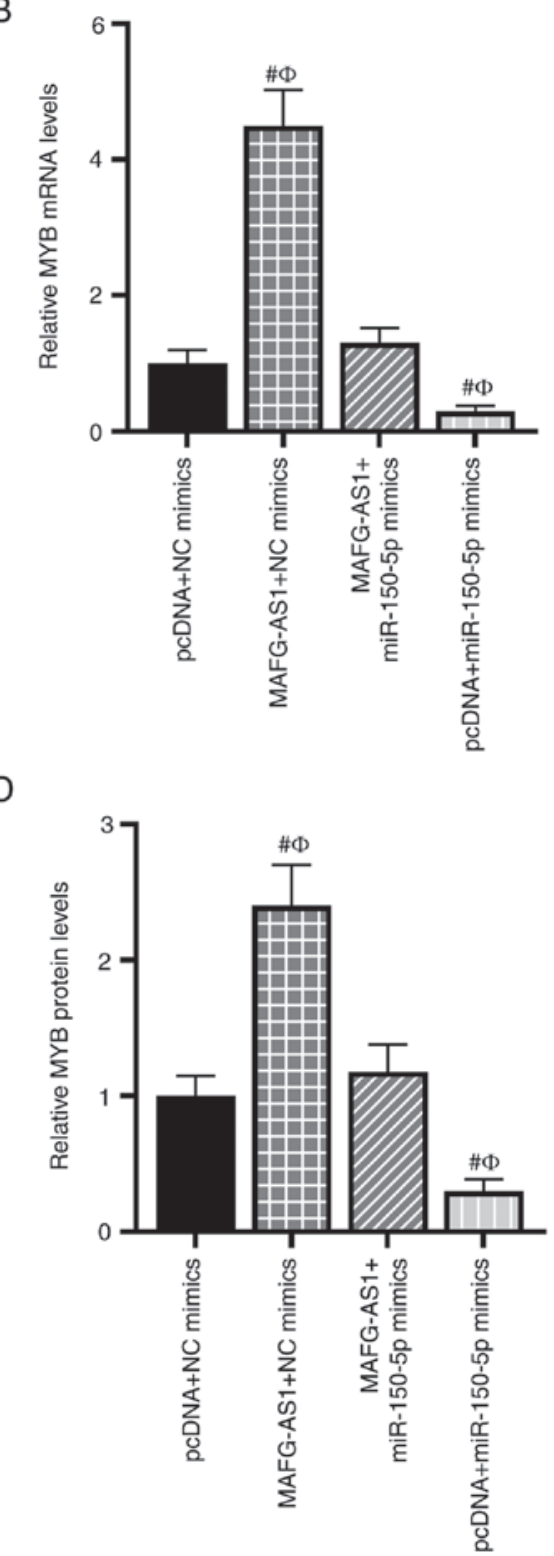

Figure 5. MAFG-AS1 positively regulates MYB expression by sponging miR-150-5p. (A) Luciferase assay was used to detect the luciferase activity in each group of cells. (B) Reverse transcription-quantitative PCR was used to detect the effects of miR-150-5p and MAFG-AS1 on MYB gene expression. (C and D) Western blotting analysis of MYB protein expression of cells overexpressing MAFG-AS1 and/or miR-150-5p. ${ }^{~} \mathrm{P}<0.05$ vs. pcDNA + NC mimics; ${ }^{\Phi} \mathrm{P}<0.05$ vs. MAFG-AS1 + miR150-5p mimics. MAFG-AS1, MAF BZIP transcription factor G antisense RNA 1; miR, microRNA.

expression levels of Bcl-2 were downregulated, and those of Bax were upregulated in breast cancer cells transfected with shMAFG-AS1 compared with those in cells transfected with the control vector, whereas MYB overexpression increased the levels of Bcl-2 and inhibited the levels of Bax compared with those in the shMAFG-AS1 group (Fig. 6H and I). Overall, these results demonstrated that MAFG-AS1 may aggravate the progression of breast cancer by upregulating MYB.

$M A F G$-AS1 regulates the progression of breast cancer in vivo by targeting the miR-150-5p/MYB axis. The present study further investigated whether MAFG-AS1 regulated breast cancer tumor growth in vivo by sponging miR-150-5p. As presented in Fig. S2A, MAFG-AS1 overexpression increased the xenograft tumor size, whereas the miR-150-5p mimics reduced the tumor size in vivo; the miR-150-5p mimics abolished the MAFG-AS1 overexpression-mediated increase in tumor size. In addition, the elevated tumor volume and tumor weight induced by MAFG-AS1 overexpression were abrogated by the miR-150-5p mimics (Fig. 2B and C). By contrast, the tumor size, volume and weight suppressed by the miR-150-5p mimics were recovered by MYB overexpression (Fig. S2D-F). These results suggested that MAFG-AS1 regulated the progression of breast cancer in vivo by targeting the miR-150-5p/MYB axis.

\section{Discussion}

Breast cancer is a major health threat to women worldwide $(1,2)$. Although a number of studies have investigated the cellular and molecular mechanisms underlying the occurrence and development of breast cancer (31-33), early detection is 
A
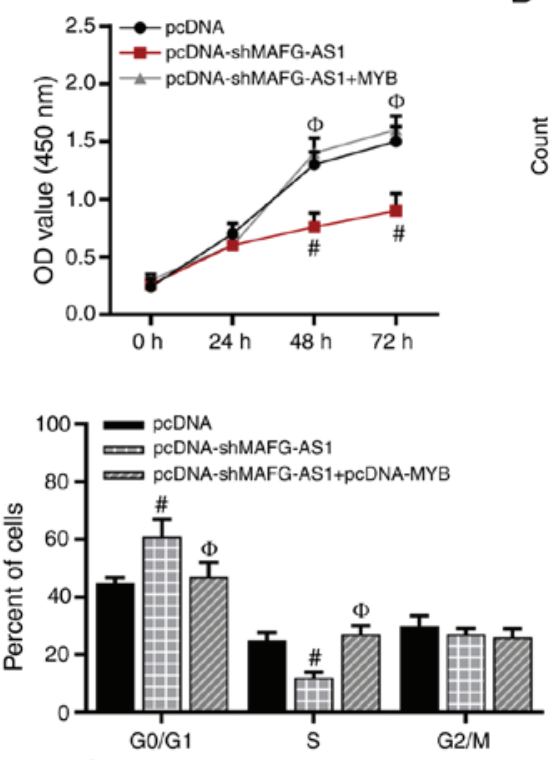

B

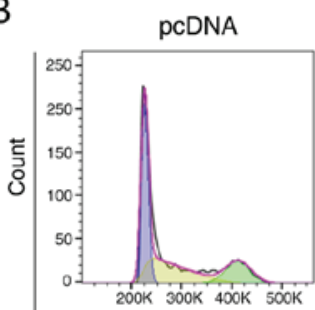

C

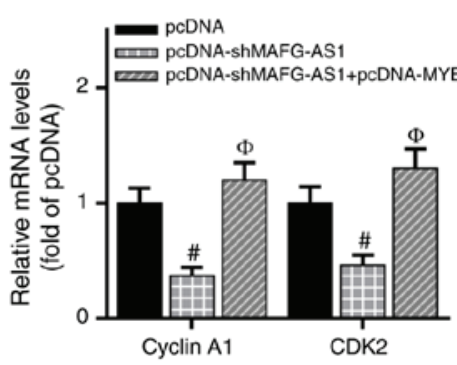

PE
pcDNA-shMAFG-AS + pcDNA-MYB
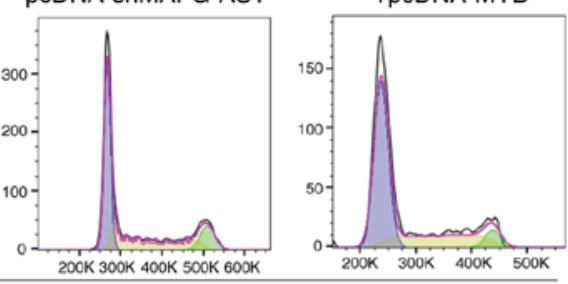

D

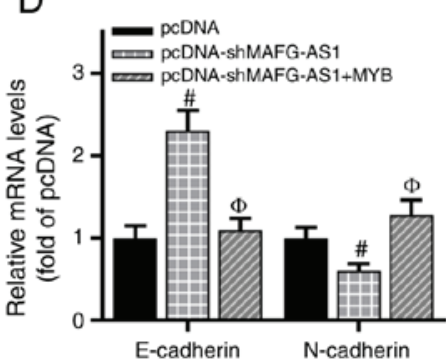

E

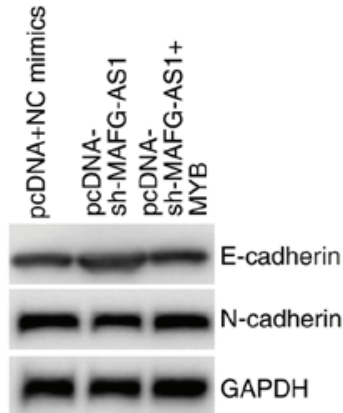

$\mathrm{G}$

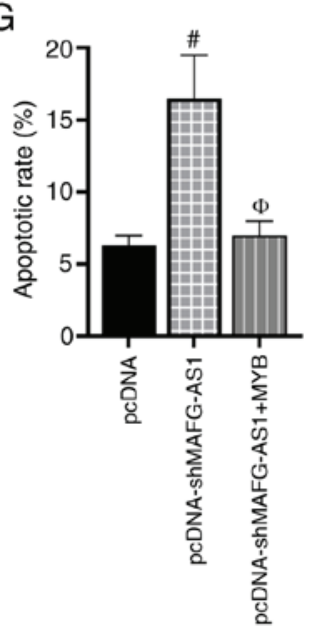

$\mathrm{F}$
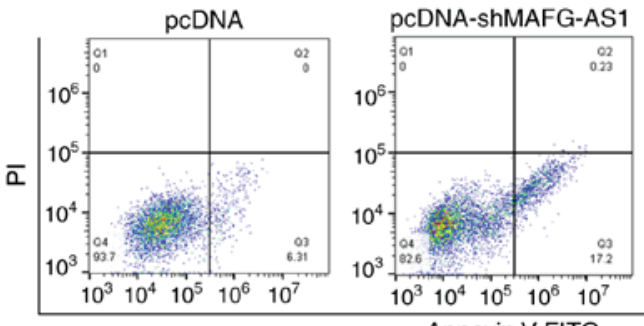

pcDNA-shMAFG-AS1+

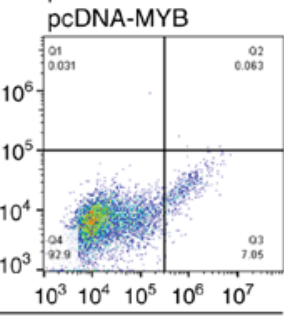

$\mathrm{H}$

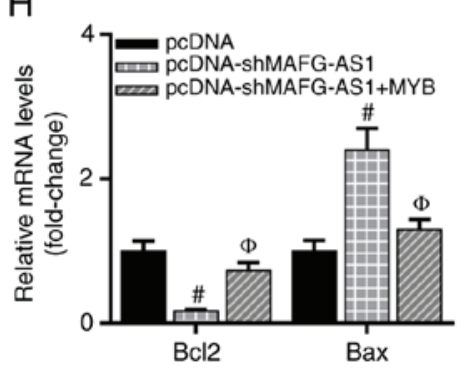

I

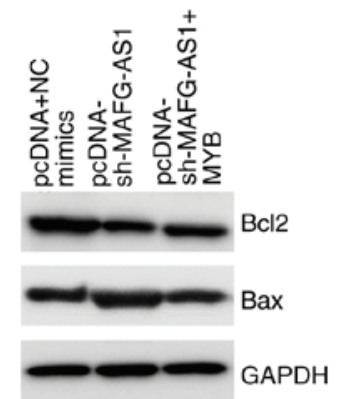

Figure 6. MYB is involved in the MAFG-AS1-mediated regulation of cell biological functions. (A) MTS was used to detect the proliferation in each group of cells. (B) The cell cycle was examined using FACS analysis. (C) RT-qPCR was used to detect the expression of the cell cycle-related genes cyclin A1 and CDK2. (D) RT-qPCR was used to detect the expression of the epithelial-mesenchymal transition-related genes E-cadherin and N-cadherin. (E) Western blotting analysis was used to detect the protein levels of E-cadherin and N-cadherin. (F and G) Annexin V-FITC was used to detect the apoptotic rates. (H) RT-qPCR was used to detect the mRNA expression levels of Bcl-2 and Bax. (I) Western blotting analysis was used to detect the protein expression levels of Bcl-2 and Bax. ${ }^{~} \mathrm{P}<0.05$ vs. pcDNA; ${ }^{\oplus} \mathrm{P}<0.05$ vs. pcDNA-shMAFG-AS1. MAFG-AS1, MAF BZIP transcription factor G antisense RNA 1; RT-qPCR, reverse transcription-quantitative PCR; sh, short hairpin RNA.

hindered by the lack of effective diagnostic biomarkers. Numerous studies have indicated that lncRNAs may be used as potential therapeutic targets or prognostic markers of breast cancer (34). For instance, lncRNA SNHG14 induces breast cancer cell chemoresistance to trastuzumab by regulating poly(A)-binding protein cytoplasmic 1 through trimethylation of lysine 27 acetylation (35). In addition, lncRNA integrin subunit $\beta 2$ (ITGB2)-AS1 promotes the migration and invasion of breast cancer cells through upregulating ITGB2 (36). However, the role of MAFG-AS1 in breast cancer has not been explored. The results of the present study demonstrated that the expression of MAFG-AS1 in breast cancer tissues 
and cells was increased compared with that in non-cancerous tissues and cells. These results indicated that MAFG-AS1 had the potential to become a biomarker for breast cancer.

The canonical theory for the mechanism of lncRNAs is that lncRNA serve as miRNA 'sponges' to antagonize the inhibition of miRNAs on mRNAs, thus enabling the expression of downstream target genes (15). For example, the lncRNA growth arrest-specific 5 regulates autophagy in patients with breast cancer by sponging miR-23a to upregulate autophagy related 3 homolog (37). IncRNA bladder cancer-associated transcript 1 induces breast cancer cell proliferative, migratory and invasive abilities by regulating $\mathrm{C}-\mathrm{C}$ chemokine receptor type 2 expression by binding to the specific sequence of miR-150-5p (15). The androgen receptor negatively induced 1 cRNA binds to miR-204 to increase the migration and invasion of triple-negative breast cancer cells (38). miRNAs serve important regulatory roles in physiological activities and pathological processes of breast cancer. miR-1287-5p suppresses the growth of triple negative breast cancer growth via interaction with phosphoinositide 3-kinase CB (39). miR-132-3p inhibits the migration and invasion of breast cells by targeting lysosomal-associated protein transmembrane 4 beta (40). The results of the present study confirmed that miR-150-5p was sponged by MAFG-AS1 in breast cancer cells. In addition, miR-150-5p was downregulated in breast cancer cells and tissues compared with normal adjacent tissues and normal breast epithelial cells. The miR-150-5p mimics blocked the progression of breast cancer and abolished the promotive role of MAFG-AS1 overexpression on the progression of breast cancer. Additionally, the in vivo experiment results demonstrated that the tumor size, volume and weight increased by MAFG-AS1 overexpression were abrogated by the miR-150-5p mimics. These results demonstrated that MAFG-AS1 promoted the progression of breast cancer by sponging miR-150-5p in vitro and in vivo.

Bioinformatics analysis in the present study demonstrated that the oncogene MYB was a candidate target gene for miR-150-5p. Previous studies have demonstrated that MYB exerts a cancer-promoting effect in a variety of tumors, including breast cancer (41-44). However, the molecular mechanism of how the oncogenic driver MYB affects breast cancer progression has not yet been fully elucidated. In the present study, the luciferase reporter assay confirmed that miR-150-5p directly targeted the 3'UTR of MYB. In addition, the miR-150-5p mimics significantly inhibited the expression of MYB in MCF-7 cells. The miR-150-5p mimics also inhibited breast cancer tumor growth in vivo by suppressing MYB expression. In addition, MYB overexpression abolished the miR-150-5p mimic-mediated inhibition of tumor size, volume and weight in vivo. Further experiments verified that MYB may be an important molecule in the MAFG-AS1-mediated regulation of breast cancer cell biological function.

In conclusion, the results of the present study revealed a crucial role of IncRNA MAFG-AS1 in the proliferation and migration of breast cancer cells. MAFG-AS1 may promote human breast cancer tumorigenesis by targeting the miR-150-5p/MYB axis, suggesting a novel molecular mechanism of breast cancer.

\section{Acknowledgements}

Not applicable.

\section{Funding}

This work was supported by the Jilin Provincial Department of Science and Technology (grant no. 20190201209JC).

\section{Availability of data and materials}

All data generated or analyzed in this study are available from the corresponding author on reasonable request.

\section{Authors' contributions}

HJ and SL conceived and designed the study. HJ, DW and ZZ performed the experiments. HJ, DW and SL analyzed the data. HJ and SL wrote the manuscript. SL edited the manuscript. All authors read and approved the final manuscript.

\section{Ethics approval and consent to participate}

The clinical study was approved by the Ethics Committee of the First Hospital of Jilin University (approval no.DWLL-2019-0021), and written informed consent was obtained from all patients. All relevant methods were performed according to the relevant guidelines and regulations. The animal study was approved with the ethical approval from the Ethics Committee of Animal Experiments of the First Hospital of Jilin University (approval no. DWLL-2019-0021).

\section{Patient consent for publication}

Not applicable.

\section{Competing interests}

The authors declare that they have no competing interests.

\section{References}

1. Ferlay J, Colombet M, Soerjomataram I, Mathers C, Parkin DM, Piñeros M, Znaor A and Bray F: Estimating the global cancer incidence and mortality in 2018: GLOBOCAN sources and methods. Int J Cancer 144: 1941-1953, 2019.

2. Bray F, Ferlay J, Soerjomataram I, Siegel RL, Torre LA and Jemal A: Global cancer statistics 2018: GLOBOCAN estimates of incidence and mortality worldwide for 36 cancers in 185 countries. CA Cancer J Clin 68: 394-424, 2018.

3. Feng RM, Zong YN, Cao SM and Xu RH: Current cancer situation in China: Good or bad news from the 2018 global cancer statistics? Cancer Commun (Lond) 39: 22, 2019.

4. de la Mare JA, Contu L, Hunter MC, Moyo B, Sterrenberg JN, Dhanani KC, Mutsvunguma LZ and Edkins AL: Breast cancer: Current developments in molecular approaches to diagnosis and treatment. Recent Pat Anticancer Drug Discov 9: 153-175, 2014.

5. Shi X, Sun M, Liu H, Yao Y and Song Y: Long non-coding RNAs: A new frontier in the study of human diseases. Cancer Lett 339: 159-166, 2013

6. Tsai MC, Spitale RC and Chang HY: Long intergenic noncoding RNAs: New links in cancer progression. Cancer Res 71: 3-7, 2011.

7. Chen X, Dai M, Zhu H, Li J, Huang Z, Liu X, Huang Y, Chen J and Dai S: Evaluation on the diagnostic and prognostic values of long non-coding RNA BLACAT1 in common types of human cancer. Mol Cancer 16: 160, 2017. 
8. Dai M, Chen X, Mo S, Li J, Huang Z, Huang S, Xu J, He B, Zou Y, Chen J and Dai S: Meta-signature LncRNAs serve as novel biomarkers for colorectal cancer: Integrated bioinformatics analysis, experimental validation and diagnostic evaluation. Sci Rep 7: 46572, 2017.

9. Ye JR, Liu L and Zheng N: Long noncoding RNA bladder cancer associated transcript 1 promotes the proliferation, migration, and invasion of nonsmall cell lung cancer through sponging miR-144. DNA Cell Biol 36: 845-852, 2017.

10. Rinn JL, Kertesz M, Wang JK, Squazzo SL, Xu X, Brugmann SA, Goodnough LH, Helms JA, Farnham PJ, Segal E and Chang HY: Functional demarcation of active and silent chromatin domains in human HOX loci by noncoding RNAs. Cell 129: 1311-1323, 2007.

11. Bhan A, Soleimani M and Mandal SS: Long noncoding RNA and cancer: A new paradigm. Cancer Res 77: 3965-3981, 2017.

12. Huang HW, Xie H, Ma X, Zhao F and Gao Y: Upregulation of LncRNA PANDAR predicts poor prognosis and promotes cell proliferation in cervical cancer. Eur Rev Med Pharmacol Sci 21: 4529-4535, 2017.

13. Zang W, Wang T, Huang J, Li M, Wang Y, Du Y, Chen X and Zhao G: Long noncoding RNA PEG10 regulates proliferation and invasion of esophageal cancer cells. Cancer Gene Ther 22: 138-144, 2015

14. Ding X, Zhang S, Li X, Feng C, Huang Q, Wang S, Wang S, $\mathrm{Xia} \mathrm{W}$, Yang F, Yin R, et al: Profiling expression of coding genes, long noncoding RNA, and circular RNA in lung adenocarcinoma by ribosomal RNA-depleted RNA sequencing. FEBS Open Bio 8: 544-555, 2018.

15. Hu X, Liu Y, Du Y, Cheng T and Xia W: Long non-coding RNA BLACAT1 promotes breast cancer cell proliferation and metastasis by miR-150-5p/CCR2. Cell Biosci 9: 14, 2019.

16. Jia YC, Wang JY, Liu YY, Li B, Guo H and Zang AM: LncRNA MAFG-AS1 facilitates the migration and invasion of NSCLC cell via sponging miR-339-5p from MMP15. Cell Biol Int 43: 384-393, 2019.

17. Cui S, Yang X, Zhang L, Zhao Y and Yan W: LncRNA MAFG-AS1 promotes the progression of colorectal cancer by sponging miR-147b and activation of NDUFA4. Biochem Biophys Res Commun 506: 251-258, 2018.

18. Li H, Zhang GY, Pan CH, Zhang XY and Su XY: LncRNA MAFG-AS1 promotes the aggressiveness of breast carcinoma through regulating miR-339-5p/MMP15. Eur Rev Med Pharmacol Sci 23: 2838-2846, 2019.

19. Wang JS, Liu QH, Cheng XH, Zhang WY and Jin YC: The long noncoding RNA ZFAS1 facilitates bladder cancer tumorigenesis by sponging miR-329. Biomed Pharmacother 103: 174-181, 2018

20. Lu S, Sun Z, Tang L and Chen L: LINC00355 promotes tumor progression in HNSCC by hindering MicroRNA-195-mediated suppression of HOXA10 expression. Mol Ther Nucleic Acids 19: 61-71, 2020.

21. Xiao H: MiR-7-5p suppresses tumor metastasis of non-small cell lung cancer by targeting NOVA2. Cell Mol Biol Lett 24: 60, 2019.

22. Zhang Q, Zheng J and Liu L: The long noncoding RNA PCGEM1 promotes cell proliferation, migration and invasion via targeting the miR-182/FBXW11 axis in cervical cancer. Cancer Cell Int 19: 304, 2019.

23. Chen X, Xu X, Pan B, Zeng K, Xu M, Liu X, He B, Pan Y, Sun H and Wang S: miR-150-5p suppresses tumor progression by targeting VEGFA in colorectal cancer. Aging (Albany NY) 10: $3421-3437,2018$

24. Lu W, Zhang H, Niu Y, Wu Y, Sun W, Li H, Kong J, Ding K, Shen HM, Wu H, et al: Long non-coding RNA linc00673 regulated non-small cell lung cancer proliferation, migration, invasion and epithelial mesenchymal transition by sponging miR-150-5p. Mol Cancer 16: 118, 2017

25. Sakr M, Takino T, Sabit H, Nakada M, Li Z and Sato H: miR-150-5p and miR-133a suppress glioma cell proliferation and migration through targeting membrane-type-1 matrix metalloproteinase. Gene 587: 155-162, 2016.

26. Edge SB and Compton CC: The American joint committee on cancer: The 7th edition of the AJCC cancer staging manual and the future of TNM. Ann Surg Oncol 17: 1471-1474, 2010

27. Livak KJ and Schmittgen TD: Analysis of relative gene expression data using real-time quantitative PCR and the 2(-Delta Delta C(T)) method. Methods 25: 402-408, 2001
28. Li JH, Liu S, Zhou H, Qu LH and Yang JH: starBase v2.0 Decoding miRNA-ceRNA, miRNA-ncRNA and protein-RNA interaction networks from large-scale CLIP-Seq data. Nucleic Acids Res 42: D92-D97, 2014.

29. Sen R, Ghosal S, Das S, Balti S and Chakrabarti J: Competing endogenous RNA: The key to posttranscriptional regulation. ScientificWorldJournal 2014: 896206, 2014.

30. Tay Y, Rinn J and Pandolfi PP: The multilayered complexity of ceRNA crosstalk and competition. Nature 505: 344-352, 2014.

31. Khatib A, Solaimuthu B, Yosef MB, Rmaileh AA, Tanna M, Oren G, Frisch MS, Axelrod JH, Lichtenstein M and Shaul YD: The glutathione peroxidase 8 (GPX8)/IL-6/STAT3 axis is essential in maintaining an aggressive breast cancer phenotype. Proc Natl Acad Sci USA 117: 21420-21431, 2020.

32. Cataldo A, Romero-Cordoba S, Plantamura I, Cosentino G, Hidalgo-Miranda A, Tagliabue E and Iorio MV: MiR-302b as a combinatorial therapeutic approach to improve cisplatin chemotherapy efficacy in human triple-negative breast cancer. Cancers (Basel) 12: 2261, 2020.

33. Naik SK, Lam EWF, Parija M, Prakash S, Jiramongkol Y, Adhya AK, Parida DK and Mishra SK: NEDDylation negatively regulates ERR $\beta$ expression to promote breast cancer tumorigenesis and progression. Cell Death Dis 11: 703, 2020.

34. Rodríguez Bautista R, Ortega Gómez A, Hidalgo Miranda A, Zentella Dehesa A, Villarreal-Garza C, Ávila-Moreno F and Arrieta O: Long non-coding RNAs: Implications in targeted diagnoses, prognosis, and improved therapeutic strategies in human non- and triple-negative breast cancer. Clin Epigenetics 10: 88, 2018.

35. Dong H, Wang W, Mo S, Liu Q, Chen X, Chen R, Zhang Y, Zou K, Ye M, He X, et al: Long non-coding RNA SNHG14 induces trastuzumab resistance of breast cancer via regulating PABPC1 expression through H3K27 acetylation. J Cell Mol Med 22: 4935-4947, 2018

36. Liu M, Gou L, Xia J, Wan Q, Jiang Y, Sun S, Tang M, He T and Zhang Y: LncRNA ITGB2-AS1 could promote the migration and invasion of breast cancer cells through up-regulating ITGB2. Int J Mol Sci 19: 1866, 2018.

37. Gu J, Wang Y, Wang X, Zhou D, Wang X, Zhou M and He Z: Effect of the LncRNA GAS5-MiR-23a-ATG3 axis in regulating autophagy in patients with breast cancer. Cell Physiol Biochem 48: 194-207, 2018.

38. Yang F, Shen Y, Zhang W, Jin J, Huang D, Fang H, Ji W, Shi Y, Tang L, Chen W, et al: An androgen receptor negatively induced long non-coding RNA ARNILA binding to miR-204 promotes the invasion and metastasis of triple-negative breast cancer. Cell Death Differ 25: 2209-2220, 2018.

39. Schwarzenbacher D, Klec C, Pasculli B, Cerk S, Rinner B, Karbiener M, Ivan C, Barbano R, Ling $\mathrm{H}$, Wulf-Goldenberg A, et al: MiR-1287-5p inhibits triple negative breast cancer growth by interaction with phosphoinositide 3-kinase CB, thereby sensitizing cells for PI3Kinase inhibitors. Breast Cancer Res 21: 20, 2019.

40. Li S, Xu JJ and Zhang QY: MicroRNA-132-3p inhibits tumor malignant progression by regulating lysosomal-associated protein transmembrane 4 beta in breast cancer. Cancer Sci 110: 3098-3109, 2019.

41. Rettig EM, Tan M, Ling S, Yonescu R, Bishop JA, Fakhry C and Ha PK: MYB rearrangement and clinicopathologic characteristics in head and neck adenoid cystic carcinoma. Laryngoscope 125: E292-E299, 2015.

42. West RB, Kong C, Clarke N, Gilks T, Lipsick JS, Cao H, Kwok S, Montgomery KD, Varma S and Le QT: MYB expression and translocation in adenoid cystic carcinomas and other salivary gland tumors with clinicopathologic correlation. Am J Surg Pathol 35: 92-99, 2011.

43. Andersson MK, Afshari MK, Andrén Y, Wick MJ and Stenman G: Targeting the oncogenic transcriptional regulator MYB in adenoid cystic carcinoma by inhibition of IGF1R/AKT signaling. J Natl Cancer Inst 109: 2017.

44. Mitra P: Transcription regulation of MYB: A potential and novel therapeutic target in cancer. Ann Transl Med 6: 443, 2018.

c) (i) $\ominus$ This work is licensed under a Creative Commons Attribution-NonCommercial-NoDerivatives 4.0 International (CC BY-NC-ND 4.0) License. 